\title{
The Bacillus Virulome in Endophthalmitis
}

3 Phillip S. Coburn ${ }^{\mathrm{a} \#}$, Frederick C. Miller ${ }^{\mathrm{b}, \mathrm{c}}$, Morgan A. Enty ${ }^{\mathrm{a}}$, Craig Land ${ }^{\mathrm{a}}$, Austin L. LaGrow ${ }^{\mathrm{a}}$,

4 Md Huzzatul Mursalin ${ }^{\mathrm{d}}$, and Michelle C. Callegan ${ }^{\mathrm{a}, \mathrm{d}, \mathrm{e}}$

6 a Department of Ophthalmology, University of Oklahoma Health Sciences Center, Oklahoma

7 City, Oklahoma, USA

$8{ }^{\mathrm{b}}$ Department of Cell Biology, University of Oklahoma Health Sciences Center, Oklahoma City,

9 Oklahoma, USA

10 cDepartment of Family and Preventive Medicine, University of Oklahoma Health Sciences

11 Center, Oklahoma City, Oklahoma, USA

12 Department of Microbiology and Immunology, University of Oklahoma Health Sciences

13 Center, Oklahoma City, Oklahoma, USA

14 e Dean McGee Eye Institute, Oklahoma City, Oklahoma, USA

16 \#Corresponding author: Phillip S. Coburn, phillip-coburn@ ouhsc.edu.

18 Key words: Bacillus cereus, endophthalmitis, ocular infections, virulence determinants, in vivo

19 virulence gene expression

21 Abbreviations: BHI, Brain Heart Infusion; RNA-Seq, RNA Sequencing; POE, post-operative

22 endophthalmitis; PTE, post-traumatic endophthalmitis; EE, endogenous endophthalmitis; PC-

23 PLC, phosphatidylcholine-specific phospholipase C; PI-PLC, phosphatidylinositol-specific 
24 phospholipase C; TLR, Toll-like receptor; Hbl, hemolysin BL; Nhe, nonhemolytic enterotoxin;

25 Ent, enterotoxin; InhA, immune inhibitor A; CerO, cereolysin O; HylA, hemolysin A; SOD,

26 superoxide dismutase; Slp, surface layer protein; RPKM, reads per kilobase million; bp,

27 basepairs. 


\section{Abstract}

Bacillus cereus is recognized as a causative agent of gastrointestinal syndromes, but can also cause a devastating form of intraocular infection known as endophthalmitis. We have

31 previously reported that the PlcR/PapR master virulence factor regulator system regulates

32 intraocular virulence, and that the S-layer protein (SlpA) contributes to the severity of B. cereus

33 endophthalmitis. To begin to better understand the role of other $B$. cereus virulence genes in

34 endophthalmitis, expression levels of a subset of factors was measured at the midpoint of disease

35 progression in a murine model of experimental endophthalmitis by RNA-Seq. Several cytolytic

36 toxins were expressed at significantly higher levels in vivo than in BHI. The virulence regulators

$37 \operatorname{cod} Y, g n t R$, and $n p r R$ were also expressed in vivo. However, at this timepoint, plcR/papR was not

38 detectable, we previously reported that a B. cereus mutant deficient in PlcR was attenuated in the

39 eye. The motility-related genes $f l a, f l i F$, and $m o t B$, and the chemotaxis-related gene cheA were

40 detected during infection. We have shown previously that motility and chemotaxis phenotypes

41 are important in $B$. cereus endophthalmitis. The sodA2 variant of manganese superoxide

42 dismutase was the most highly expression gene in vivo, suggesting that this gene is criticial for

43 intraocular survival, potentially through inhibition of neutrophil activity. Expression of the

44 surface layer protein gene, $\operatorname{slpA}$, an activator of Toll-like receptors (TLR)-2 and -4, and a potent

45 contributor to intraocular inflammation and disease severvity, was also detected during infection,

46 albeit at low levels. In summary, genes expressed in a mouse model of Bacillus endophthalmitis

47 might prove to play crucial roles in the unique virulence of $B$. cereus endophthalmitis, and serve

48 as candidates for novel therapies designed attenuate the severity of this often blinding infection.

\section{Impact statement}


B. cereus causes a potent and rapid infection of the eye that usually results in blindness or

52 enucleation, even with the utilization of current treatment modalities. This necessitates the

53 development of new treatment modalities based on new targets. To begin to better define those

54 B. cereus factors with roles in intraocular infection, we analyzed the expression of genes with

55 both known and hypothesized roles in intraocular infection at the midpoint of infection using a

56 murine model of Bacillus endophthalmitis. Potentially targetable candidate genes were

57 demonstrated to be expressed in vivo, which suggests that these genes might contribute to the

58 unique virulence of $B$. cereus endophthalmitis. Importantly, our results begin to define the

59 virulome of $B$. cereus in intraocular infections and identify previously uncharacterized factors

60 with potential roles in the severity and outcome of Bacillus endophthalmitis.

\section{Introduction}

Endophthalmitis is an infection of the anterior and posterior segments of the eye

64 following the introduction of microorganisms after a surgical procedure (post-operative

65 endophthalmitis [POE]), a traumatic penetrating injury (post-traumatic endophthalmitis [PTE]),

66 or hematogenous spread from an infection of a distant site in the body (endogenous

67 endophthalmitis [EE]) [1-5]. Intraocular infection with B. cereus results in devastating

68 inflammation of the eye and, in an unfortunately large number of cases, blindness. Bacillus

69 endophthalmitis is one of the most rapidly evolving and severe forms of this infection, and is

70 characterized by fulminant intraocular inflammation and significant vision loss within hours [1-

71 5]. Severe pain, periorbital edema, corneal ring abscesses, anterior chamber inflammation,

72 vitritis, a decreased fundus reflex, propotosis, and a rapid decline in visual acuity are common

73 symptoms of the disease. The destruction of the ocular and retinal architecture has been 
74 postulated to result from both bacterial- and host immune-mediated mechanisms. Approximately

$7570 \%$ of patients with Bacillus endophthalmitis suffer a significant loss in visual acuity, and half

76 of these require surgical removal of the eye, even with aggressive therapeutic intervention [1-5].

77 Currently, no universally successful therapeutic regimen has been described to treat this

78 infection. Ineffective antibiotic penetration and contradictory clinical reports on the dose, route,

79 and combination therapy make treating endophthalmitis difficult [1-5]. Moreover, the clinical

80 outcome of this disease is influenced by age, extent of injury, and delays in the initiation of

81 treatment. Examining the mechanisms of both bacterial and host factors in the pathogenesis of $B$.

82 cereus endophthalmitis is of utmost importance in order to develop better therapeutic strategies

83 to treat this blinding disease.

84 B. cereus toxins and cell wall components cause damage to the nonregenerative tissues of

85 the eye during endophthalmitis, either through direct effects on retinal cells or indirectly due to

86 bystander damage from immune cell responses [6-10]. We demonstrated that the PlcR/PapR

87 system, the major transcriptional regulatory system of toxins and virulence in B. cereus,

88 contributes to intraocular virulence [9]. However, we could not ascribe individual roles for

89 hemolysin BL, phosphatidylcholine-specific phospholipase C (PC-PLC), and

90 phosphatidylinositol-specific phospholipase C (PI-PLC) in a rabbit model of endophthalmitis [7,

91 11]. The role of other toxins elaborated by B. cereus, including cereolysin O, cytotoxin $\mathrm{K}$, and

92 nonhemolytic enterotoxin (Nhe), have not yet been investigated.

93 The cell wall of B. cereus was more inflammogenic than the cell walls of the intraocular

94 pathogens S. aureus and E. faecalis in a rabbit model of endophthalmitis [12]. Based on these

95 results, we hypothesized that $B$. cereus cell walls possess a unique structural feature and/or a

96 common component that has evolved structural differences from other Gram-positive pathogens. 
97 A thick peptidoglycan layer, capsular polysaccharide, lipotechoic and teichoic acids,

98 lipoproteins, pili, a glycoprotein S-layer, and flagella comprise the B. cereus cell wall [13-15]. B.

99 cereus pili interfered with bacterial clearance in a mouse model of endophthalmitis [16],

100 suggesting a role as an antiphagoctyic factor. In the same model, a mutant strain of Bacillus

101 deficient in the S-layer protein, SlpA, was less virulent relative to the wild type, parental strain

102 [17]. Moreover, Bacillus SlpA stimulated nuclear factor kappa-light-chain-enhancer of activated

$103 \mathrm{~B}$ cells $(\mathrm{NF}-\kappa \mathrm{B})$ in human retinal Muller cells in vitro, interfered with phagocytosis by

104 neutrophils and retinal cells, and served as an adhesin to retinal cells [18]. Bacillus flagella

105 contribute to the intraocular virulence of B. cereus by facilitating migration throughout the eye

106 [12]. However, mutants defective in motility resulted in only a delay in disease progression [8,

107 19].

We previously reported that $B$. cereus known and putative virulence determinants,

109 virulence-related transcriptional regulators, and superoxide dismutase were expressed in ex vivo

110 rabbit vitreous and suggested that these genes might contribute to the unique virulence of $B$.

111 cereus in the eye. In the current study, expression levels of factors known or hypothesized to be

112 involved in virulence and transcriptional regulation, motility, and chemotaxis were measured at

113 the midpoint of infection progression (8 hours postinfection) in an in vivo murine model of $B$.

114 cereus endophthalmitis. We also evaluated the expression of these genes after growth in Brain

115 Heart Infusion (BHI) broth at the same time point. The aim of this study was to identify factors

116 expressed in vivo that might contribute to pathogenesis and disease progression, and potentially

117 serve as targetable candidates for novel therapeutics. At 8 hours postinfection, significant

118 bacterial growth, inflammation, and retinal function loss are observed in this model. We

119 observed that cytolytic enterotoxins, hemolysins, immune inhibitor metalloproteases, and 
120 superoxide dismutases were among the most highly expressed genes in the eye at this time point.

121 These results demonstrated the expression of both putative and known B. cereus virulence genes

122 during the midpoint of experimental murine endophthalmitis, and suggested that evaluation of

123 the dynamic virulome during endophthalmitis progression might identify possible targetable

124 candidate genes for novel therapies that aim to reduce virulence.

Methods

Bacterial strain and analysis of growth in BHI. Vegetative B. cereus ATCC 14579 was

128 cultivated in Luria Burtani (LB) for $18 \mathrm{~h}$ at $37^{\circ} \mathrm{C}$. The culture was centrifuged for 10 minutes at

$1294,300 \mathrm{x}$ g, and the bacterial pellet washed 3 times with sterile phosphate-buffered saline $(\mathrm{PBS} \mathrm{pH}$

130 7.4) to remove all traces of LB. After the third wash, the bacterial pellet was resuspended in an

131 equal volume of PBS as the original culture volume and diluted to $200 \mathrm{CFU} / \mu \mathrm{l}$ in PBS prior to

132 intravitreal injection. To assess growth in BHI, bacteria in PBS were diluted to $10^{3} \mathrm{CFU} / \mathrm{ml}$ in

133 freshly prepared BHI, and at 8 hours following back-dilution, $20 \mu 1$ aliquots were diluted 10-fold

134 in sterile PBS. Aliquots from each dilution were plated onto BHI agar plates for bacterial

135 quantification. Bacterial concentrations in $\mathrm{CFU} / \mathrm{ml}$ were determined and multiplied by $0.4 \mathrm{ml}$ to

136 determine the bacterial concentration in $400 \mu 1$ of BHI, the volume of PBS used for

137 homogenization of B. cereus-infected eyes. Two separate growth analysis experiments were

138 performed using independent batches of BHI, and each experiment was performed with 3

139 independent cultures. The mean \pm the standard error of the mean of the 6 independent cultures is

140 shown.

141 Murine endophthalmitis model. This study was conducted in accordance with the

142 recommendations in the Guide for the Care and Use of Laboratory Animals of the National 
143 Institutes of Health. The animal use protocol was approved by the Institutional Animal Care and

144 Use Committee of the University of Oklahoma Health Sciences Center (protocol number 18-

145 043). Six week old C57BL/6J mice were acquired from the Jackson Laboratory (Catalog 000664,

146 Bar Harbor ME). Mice were acclimated to conventional housing one week prior to injection to

147 allow for physiological and nutritional stabilization and to equilibrate their microbiota. All mice

148 were housed in microisolation conditions on a $12 \mathrm{~h}$ on/12 $\mathrm{h}$ off light cycle prior to the

149 experiments under biosafety level 2 conditions during experiments. Mice were 8-10 weeks of

150 age at the time of the experiments.

151 Mice were anesthetized with a combination of ketamine $(85 \mathrm{mg} / \mathrm{kg}$ body weight;

152 Ketathesia, Henry Schein Animal Health, Dublin, $\mathrm{OH})$ and xylazine (14 mg/kg body weight;

153 AnaSed; Akorn Inc., Decatur, IL). Intravitreal injections were performed with sterile borosilicate

154 glass micropipettes (Kimble Glass Inc, Vineland, NJ, USA) beveled to an approximate bore size

155 of 10 to $20 \mu \mathrm{m}$ (BV-10 KT Brown Type micropipette beveller, Sutter Instrument Co., Novato,

156 CA, USA). Eyes were visualized with a stereomicroscope and the micropipettes were inserted

157 just posterior to the superior limbus. B. cereus ATCC 14579 at a concentration of $100 \mathrm{CFU}$ in

$158 \quad 0.5 \mu \mathrm{l}$ was injected into the right eyes of 25 mice. Injection rates and volumes were monitored

159 using a programmable cell microinjector (Microdata Instruments, Plainfield, NJ, USA).

160 Triplicate independent experiments consisting of 25 mice per experiment were performed.

In vivo bacterial quantitation. For each in vivo experiment, at 8 hours postinfection, all

162 mice were euthanized by $\mathrm{CO}_{2}$ asphyxiation. Eyes were removed, and 5 eyes were placed into

163 each of 5 separate tubes containing $400 \mu \mathrm{l}$ of sterile PBS and $1.0 \mathrm{~mm}$ sterile glass beads (Biospec

164 Products Inc., Bartlesville, OK), and homogenized for 60 seconds at 5,000 rpm in a Mini-

165 BeadBeater (Biospec Products, Inc., Bartlesville, OK). Aliquots from each tube were serially 
166

167

168

169

170

171

172

173

174

175

176

177

178

179

180

181

182

183

184

185

186

187

diluted and plated on $\mathrm{BHI}$ agar plates, and incubated at $37^{\circ} \mathrm{C}$. After overnight incubation, the CFU per eye for each tube was calculated by determining the total CFU in $400 \mu \mathrm{l}$ and dividing by 5 . The value shown represents the mean \pm standard error of the mean of the CFU/eye from the 5 tubes and 3 independent experiments.

RNA preparation and quantitative PCR Analysis. For the in vitro BHI experiments, bacterial cultures were centrifuged for 10 minutes at 4,300 $\mathrm{x}$ g and the bacterial cell pellet resuspended in the lysis buffer (RLT) from the RNeasy kit (Qiagen, Germantown, MD). Bacterial cells were then homogenized with sterile $0.1 \mathrm{~mm}$ glass beads (Biospec Products Inc., Bartlesville, OK) for 60 seconds at 5,000 rpm in a Mini-BeadBeater (Biospec Products Inc., Bartlesville, OK). Total RNA was purified using the RNeasy kit according to the manufacturer's instructions (Qiagen). Genomic DNA was removed using the TURBO DNA-free kit (ThermoFisher Scientific, Inc., Waltham, Massachusetts), and ribosomal RNA was depleted using the Ribo-Zero rRNA Removal kit for bacterial rRNA (Illumina, San Diego, CA) according to the manufacturers' instructions. For total bacterial RNA isolation from B. cereus-infected eyes, 5 separate tubes, each containing 5 infected eyes in $400 \mu 1$ of sterile PBS and $1.0 \mathrm{~mm}$ sterile glass beads (Biospec Products Inc., Bartlesville, OK), were homogenized for 60 seconds at 5,000 rpm in a Mini-BeadBeater (Biospec Products, Inc., Bartlesville, OK). Homogenates were centrifuged at $500 \mathrm{x}$ g for 5 minutes to pellet ocular debris, and the supernatants were centrifuged for 10 minutes at 4,300 x g to pellet bacteria. Bacterial pellets were then resuspended in RLT buffer from the RNeasy kit (Qiagen, Germantown, MD), and transferred to tubes with sterile $0.1 \mathrm{~mm}$ glass beads (Biospec Products Inc., Bartlesville, OK). Bacteria were homogenized for 60 seconds at 5,000 rpm in a Mini-BeadBeater (Biospec Products Inc., Bartlesville, OK), and bacterial RNA was then purified using RNeasy kit (Qiagen, Germantown, MD). Purified total 
189 bacterial RNA from each of the 5 tubes was pooled and genomic DNA removed using the

190 TURBO DNA-free kit (ThermoFisher Scientific, Inc., Waltham, Massachusetts). Ribosomal

191 RNA was depleted using the Ribo-Zero rRNA Removal kit for bacterial rRNA (Illumina, San

192 Diego, CA), and depletion was confirmed via quantitative PCR using primers specific to the $B$.

193 cereus 16 s ribosomal RNA as previously described [20].

RNA sequencing. The triplicate enriched RNA samples obtained from the in vitro BHI and the in vivo experiments was sequenced using an Illumina MiSeq Next Generation Sequencer

196 at the OUHSC Laboratory for Molecular Biology and Cytometry Research. Raw data for each

197 sample was analyzed using the CLC Genomics Workbench software (Qiagen, Redwood City,

198 CA). Raw sequence reads were mapped to the $B$. cereus ATCC 14579 reference genome for

199 identification of the genes of interest in our study expressed after growth in BHI or in vivo.

200 Reads not mapped were excluded further analysis. The number of reads per gene was normalized

201 according to the total number of reads in each library and the gene size. The resulting number

202 was expressed as normalized reads per kilobase per million (RPKM). The values represent the

203 mean RPKM \pm the standard deviation of the three independent sequencing runs for each

204 condition.

Statistics. For in vitro growth in BHI, the data is the arithmetic mean \pm the standard error

207 replicates per experiment. For the in vivo growth experiments, the data is the mean \pm standard

208 error of the mean of the CFU/eye from the 5 tubes and 3 independent experiments. For RNA-

209 Seq, the data are the arithmetic means \pm the standard deviations of the RPKM values of each

210 gene derived from triplicate in vitro $\mathrm{BHI}$ and in vivo samples. Comparative differences between

211 groups were taken to be statistically significant when $\mathrm{p}<0.05$. The two-tailed, unpaired t-test 
212 with Welch's correction was used to compare the growth analysis and the RPKM values of the

213 genes in each condition. All statistical analyses were performed using GraphPad Prism 8.2.0

214 (GraphPad Software, Inc., La Jolla CA).

215 Data availability. The RNA-Seq data will be deposited in the Sequence Read Archive at

216 NCBI upon acceptance for publication.

\section{Results}

Growth of B. cereus in BHI and in vivo. In vitro growth of B. cereus ATCC 14579 in

220 BHI 8 hours after back-dilution was compared to in vivo growth 8 hours postinfection. As shown

221 in Fig.1, a statistically significant difference was observed in growth between BHI and in the

222 mouse eye $(\mathrm{p}=0.0038)$. These results demonstrate that the total CFU in $400 \mu \mathrm{l}$ of BHI was

223 approximately a half an order of magnitude higher than the CFU in each eye homogenized in 400 $224 \mu 1$ of PBS.

226 study, expression levels of factors known and hypothesized to be involved in B. cereus

227 endophthalmitis were evaluated during the midpoint of infection in our murine model of

228 endophthalmitis using RNA-Seq. B. cereus ATCC 14579 was utilized in this studies since it

229 represents a prototypical strain with a sequenced and annotated genome. The virulence and

230 pathogenesis in endophthalmitis has been previously evaluated and established [9, 16, 21-23].

231 Similar to our previous results [20], quantitative PCR analysis using primers complementary to

232 the B. cereus ATCC 14579 16s rRNA gene demonstrated that the rRNA from the in vitro BHI-

233 derived and in vivo mouse eye-derived bacterial RNA was depleted (data not shown). 
The $B$. cereus ATCC 14579 genome is 5,411,809 bp, and consists of 5,473 genes. The

235 mean read length was $151 \mathrm{bp}$ for all BHI and in vivo samples. The mean number of mapped

236 reads and mean percentage of reads that mapped to the B. cereus genome for the BHI and in vivo

237 samples is shown in Table 1. The mean number of mapped reads for the three BHI-derived RNA

238 samples was $25,505,762$ with a mean of $96 \%$ of the reads mapping to the $B$. cereus 14579

239 genome. The mean number of mapped reads for the in vivo-derived RNA samples was

$24012,360,200$, and a mean of $99 \%$ of the reads mapped to the genome.

242 a subset of known and hypothesized virulence genes, transcriptional regulators of virulence-

243 related genes, and genes related to motility and chemotaxis that we previously analyzed in vitro

244 and in ex vivo vitreous [20]. Expression levels of genes encoding the toxin hemolysin BL (Hbl),

245 the nonhemolytic enterotoxin Nhe, the putative enterotoxins EntA and EntC, entertoxin FM, the

246 putative hemolysin A, cereolysin O, and the metalloproteases InhA1, InhA2, InhA3, and

247 camelysin were measured. The transcriptional regulatory systems related to virulence (SinR/SinI,

248 EntD, CodY, GntR, NprR, and PlcR/PapR), motility-related proteins Fla, FliF, and MotB, and

249 chemotaxis-associated proteins CheA, CheR, and CheY were also evaluated. Superoxide

250 dismutase expression levels in vivo were also measured, as our previous data suggested an

251 important role for this factor [20]. Finally, the expression of the surface layer protein gene $(\operatorname{slp} A)$

252 was evaluated as this protein is an important contributor to activation of TLR-2 and -4 receptors

253 and initiation of the inflammatory response $[18,24]$. Principal component analysis of the RPKM

254 values for each of these mRNAs isolated from B. cereus after 8 hours of growth in BHI or 8

255 hours after intravitreal injection was performed. Fig. 2 shows that the three BHI and in vivo 
replicates clustered into distinct gene expression patterns. This suggested reproducible results

257

258

259

260

261

262

263

264

265

266

267

268

269

270

271

272

273

274

275

276

277

278 among the replicates of each condition.

Toxin expression at 8 hours postinfection was observed at levels similar or higher than in BHI. Among those that were expressed in vivo were Hbl, Nhe, EntA, EntC,EntFM HlyA, CerO, InhA1, and InhA2. Mean RPKM values of $h b l L 1, h b l L 2$, and $h b l B$ in vivo were 744,1002 , and 1543, respectively (Fig. 3A). Expression of these genes was 21-, 36-, and 38-fold higher in vivo than in BHI ( $\mathrm{p} \leq 0.0173)$, respectively. Mean RPKM of the nheL1 and nheL2 genes was 1694 and 410, respectively (Fig. 3B). Expression of nheL1 was 26-fold ( $\mathrm{p}<0.0001)$ and nheL2 was 7-fold $(\mathrm{p}=0.0628$ ) higher in vivo than in BHI, with the latter not being significant. Mean RPKM values of 1182 for entA, and 1533 for entC were observed in vivo (Fig. 3C). Expression of entA was significantly higher in vivo than in $\mathrm{BHI}(\mathrm{p}=0.0068)$, however entC expression was not significantly different in vivo than in $\mathrm{BHI}(\mathrm{p}=0.1827)$. The entFM gene was expressed at similar levels in vivo (mean RPKM = 1503) and BHI (mean RPKM = 1839) $(\mathrm{p}=0.6257)$ (Fig. 3C). The HlyA-encoding gene was expressed at nearly identifical levels in vivo (mean RPKM = 364) and BHI (mean RPKM = 379) (p=0.8876) (Fig. 3D). The cholesterol dependent cytolysin (CDC) cereolysin $\mathrm{O}$ gene was 9-fold higher in vivo than in $\mathrm{BHI}(\mathrm{p}=0.0050)$ (Fig. 3D).

The gene expression of the metalloproteases InhA1, InhA2, InhA3, and camelysin were measured in vivo relative to BHI. The inhA1 and inhA2 genes were expressed in vivo to a similar degree, with a mean RPKM of 97 for inhA1 and 122 for inhA2 (Fig. 3E). Transcript levels of inhAl were 6-fold higher in vivo than in $\mathrm{BHI}(\mathrm{p}=0.0270)$, however, inhA2 levels were not significantly different between the environments $(\mathrm{p}=0.4350)$. In both environments, expression of $i n h A 3$ was not detected (Fig. 3E), as expected since this strain does not express inhA3 due to a mutation in $n p r$ [25]. The calY gene, which encodes a cell surface-associated metalloproteinase, 
camelysin, was also not expressed in either environment (Fig. 3E), although we previously observed expression in LB and ex vivo vitreous [20]. These results indicate that with exception of inhA3 and calY, expression of both known and putative B. cereus virulence factors occurred in vivo, with expression levels being higher than or similar to BHI. genes reported to be involved in virulence factor regulation was highly variable in BHI and at 8 hours postinfection in vivo (Fig. 4). The biofilm formation- and enterotoxin-related regulatory gene $\sin R$ was not detected at this time point in vivo (Fig. 4A) but was significantly higher in BHI $(\mathrm{p}=0.0024)$. Expression of the SinR inhibitor sinI was not detected in vivo or in BHI (Fig. 4A). Similarly, expression of entD was not detected in both environments (Fig. 4B). In BHI, $\operatorname{cod} Y$ levels were 2-fold higher than in vivo (p=0.0196) (Fig. 4B). While expression of gntR was

290 higher in vivo than in BHI, this difference was not significant ( $\mathrm{p}=0.2884$, Fig. 4C). However, 291 nprR expression was 56-fold higher in vivo relative to BHI ( $\mathrm{p}=0.0014$, Fig. 4C). Of considerable interest is that the expression of the master toxin and virulence gene regulatory system plcR/papR was not detected 8 hours postinfection (Fig. 4D). Mean RPKM values of 10 for $p l c R$

294 and 45 for papR were observed in BHI (Fig. 4E). The $p l c R$ gene expression level was not 295 significantly higher in BHI than in vivo $(\mathrm{p}=0.0619)$; however papR expression was significantly

296 higher in BHI than in vivo ( $\mathrm{p}=0.0025)$. In summary, only codY, gntR, and $n p r R$ were expressed at 2978 hours postinfection in vivo, with the regulators gntR and $n p r R$ being the most highly expressed 298 regulators in the eye.

\section{Expression of genes associated with motility and chemotaxis in vivo. B. cereus}


302

303

304

305

306

307

308

309

310

311

312

313

314

315

316

317

318

319

320

321

322

323

324

253, and the flagellin subunit gene $f l a$ was 785 (Fig. 5A). Expression of $m o t B$ and $f l i F$ were 2fold ( $\mathrm{p} \leq 0.0147)$, and fla was 7-fold ( $\mathrm{p}<0.0001)$ higher in BHI than in vivo. The mean RPKM of cheA, cheY, and cheR was 139, 0, and 0, respectively, in vivo (Fig. 5B). Expression levels of cheA, cheY, and cheR were significantly higher in BHI ( $\mathrm{p} \leq 0.0415)$. Expression levels of the motility-, but not chemotaxis-related genes in the eye correlated with motility and migration of B. cereus within the eye at this time point during endophthalmitis.

Expression of superoxide dismutase (SodA) in vivo. The genes sodA1 and sodA2, encoding two variants of manganese superoxide dismutase, were highly expressed during infection of the eye. The mean RPKM for sodA1 was 1,891 and for sodA2 was 16,721 in vivo (Fig. 6), the latter being the most highly expressed gene in vivo. Expression of sodAl in vivo was not significantly than in BHI ( $\mathrm{p}=0.4608)$, but expression of $\operatorname{sodA} 2$ was significantly higher in vivo than after growth in BHI $(\mathrm{p}=0.0460)$. The high level expression of $\operatorname{sodA} 2$ observed at 8 hours postinfection suggested that superoxide dismutase might protect B. cereus from internallyproduced superoxide anion during growth, or possibly from superoxide generated by neutrophils, the expression of which was not assessed in this study.

\section{Expression of surface layer protein (SlpA) in vivo. The Bacillus surface layer protein,}

SlpA, has been shown to play a key role in activation of both TLR-2 and -4 and in the severity of infection in a mouse model of Bacillus endophthalmitis [17, 18, 24]. The mean RPKM of slpA was 25 in vivo, and 36 after growth in BHI (Fig. 7). The means were not statistically different $(\mathrm{p}=0.3777)$. While this level is relatively low compared to the expression of other known virulence factors in vivo, detection of $\operatorname{slp} A$ transcripts during infection is consistent with our results showing that $\operatorname{slp} A$ contributes to the inflammatory response and disease severity in a mouse model of Bacillus endophthalmitis. 


\section{Discussion}

The objective of the current study was to identify known and putative B. cereus virulence factor expression in a mouse model of endophthalmitis, and compare this to expression in laboratory medium BHI. Eight hours postinfection was evaluated because we have consistently observed significant inflammatory cell influx and retinal architectural damage at this time point. In both rabbit and mouse endophthalmitis models, B. cereus replication upon entry into the vitreous and dissemination throughout the globe occurs rapidly [12, 17, 23]. As early as 4 hours postinfection, inflammatory cells (primarily neutrophils) enter the eye but fail to control the growth of B. cereus. At 12 to 18 hours following bacterial entry, a complete dissolution of the

334 retinal architecture and retinal function loss is observed [23]. B. cereus endophthalmitis in 335 human patients also occurs following a similarly rapid course, and results in loss of vision or 336 complete loss of the globe in 12 to 48 hours [2-5]. Evaluation of the virulome of B. cereus in

337 vivo might lead to the identification of B. cereus factors that contribute to immune cell 338 interference and pathogenesis. $\mathrm{Hbl}$ and Nhe are both pore-forming toxins regulated by the PlcR/PapR system, and have

340 known roles in B. cereus gastrointestinal infections. Both sets of toxin genes were expressed at 8

341 hours postinfection in our murine model of B. cereus endophthalmitis. Two lytic components, L1

$342(h b l D)$ and L2 ( $h b l C)$, and a single binding component, B ( $h b l A)$ comprise the secreted,

343 hemolytic $\mathrm{Hbl}$ toxin [26, 27]. Hbl toxin expression was observed among subsets of isolates that

344 caused gastrointestinal infections [28, 29], and the hblCA genes were detected in endophthalmitis

345 and keratitis isolates by PCR [30]. In a rabbit model of B. cereus endophthalmitis, no difference

346 in infection progression was observed between a wild type B. cereus and an isogenic Hbl-

347 deficient mutant [11]. However, injection of purified Hbl into rabbit eyes resulted in pathological 
348 effects similar to those observed during infection with live bacteria [27]. Taken together, these

349 results suggested that additional virulence factors might contribute to retinal damage and the

350 unique inflammatory response to B. cereus infection. Here, Hbl expression was significantly

351 higher at 8 hours postinfection than in BHI. We cannot rule out that cytotoxicity in vivo might be

352 attributable to a combination of B. cereus secreted toxins. Nhe is also a tripartite toxin consisting

353 of the lytic components NheA (nheL2) and NheB (nheL1), and the binding component NheC

354 [31]. Nhe was shown to be expressed in the majority of gastrointestinal-associated isolates [28,

355 29]. We previously observed lower level expression of these toxins in ex vivo vitreous than in

356 laboratory media [20]. In the current analysis, $\mathrm{Hbl}$ and Nhe were expressed at significantly

357 higher levels at 8 hours postinfection, suggesting that the in vivo environment influences

358 expression of these genes.

We also evaluated the expression of the putative cell wall peptidase-encoding gene

$360 e n t F M$, and two putative enterotoxin/cell-wall binding protein genes entA, and entC at the

361 midpoint of endophthalmitis progression. Similar levels of the $e n t F M$ and entC genes were

362 detected in vivo and after 8 hours of growth in BHI. However, expression of entA was higher at

363 the midpoint of infection than after growth in BHI. EntFM contributed to vacuolization in

364 macrophages, in vitro adhesion and biofilm formation, and lethality in a Galleria mellonella

365 model [32], it is not clear whether EntA or EntC contribute to virulence in any model.

Expression of the HlyA-encoding gene hlyA was similar in vivo and in BHI.However the

367 cereolysin O-encoding gene cerO was expressed at significantly higher levels in vivo than in

368 BHI. Cereolysin $\mathrm{O}(\mathrm{CerO})$ is a member of the cholesterol-dependent cytolysin (CDC) family of

369 pore-forming toxins. At 4 hours postinfection with a CerO-deficient B. cereus strain, TLR4-

370 dependent inflammatory mediators were significantly downregulated [33]. TLR4 contributed to 
371 the inflammatory response to B. cereus [22], and was activated by purified B. cereus SIpA

372 protein [18]. In addition to direct cellular cytotoxicity, CerO as a CDC might interact with and/or

373 activate TLR4 [34-37], inciting inflammation during B. cereus endophthalmitis.

374 In vivo, the metalloprotease genes inhA1 and inhA2 genes were expressed at similar

375 levels. Expression of inhA1 and inhA2 were also similar to one another in ex vivo vitreous [20].

376 InhAl was expressed at significantly higher levels in vivo than in BHI, however, inhA2 was

377 expressed at similar levels in both environments. We did not observe expression of inhA3 in

378 either environment, similar to our previous study that did not detect expression in BHI, LB, or ex

379 vivo vitreous [20]. These observations concur with the report that B. cereus ATCC 14579 does

380 not express inhA3 due to a mutation in $n p r$ [25]. The cell surface-associated metalloproteinase,

381 camelysin (CalY), was also not expressed in either environment, in contrast to our previous study

382 that expression was detected in LB and ex vivo vitreous [20]. The InhA metalloproteases have

383 been proposed to contribute to virulence by mediating degradation of extracellular matrix

384 components and escaping host defenses [38]. Based on these results, it might be postulated that

385 the InhA metalloproteases might damage the eye by breaking down type II collagen in the

386 vitreous [39], or by mediating escape from neutrophils. CalY degrades serum protease inhibitors,

387 collagen type I, fibrin, fibrinogen, and plasminogen [40, 41]. While we reported that calY

388 expression was similar to inhA expression after growth in the vitreous environment, calY

389 transcripts were not detected at 8 hours postinfection. However, these results do not preclude the

390 involvement of CalY in pathogenesis, as expression might occur at a different time point during

391 intraocular infection.

Among the transcriptional regulators analyzed, only $\operatorname{cod} Y$, gntR, and $n p r R$ were

393 expressed at 8 hours postinfection. gntR and $n p r R$ were the most highly expressed. CodY is a 
394 global transcriptional regulator that affects both the PlcR/PapR and Spo0A-AbrB regulatory

395 systems [42]. Deletion of $\operatorname{cod} Y$ decreased the expression of PlcR-regulated virulence genes,

396 resulting in attenuated infection in a G. mellonella model [42]. On the contrary, deletion of codY

397 in Staphylococcus aureus did not alter its intraocular growth but did result in significantly higher

398 inflammatory scores and lower retinal function retention as compared to the parental wild type

399 strain [43]. We reported that $\operatorname{cod} Y$ was expressed in ex vivo vitreous as well as in BHI and LB

400 media during stationary phase [20]. In the current study, we detected $\operatorname{cod} Y$ transcripts at the

401 midpoint of endophthalmitis. Given that CodY binds to the promoter of InhA1 [42], our results

402 suggest that CodY might play an integral role in the in vivo regulation of inhA1. Moreover,

403 CodY might activate the expression of secreted proteases involved in extracellular matrix

404 degradation and thus promote physical barrier breach in the eye. CodY might also regulate the

405 expression of metabolic pathway genes that are needed to utilize available nutrient sources

406 during intraocular infection.

407 The gluconate transcriptional repressor, GntR, was expressed in vivo at the 8-hour time

408 point. Expression was also observed after 18 hours of growth in BHI, LB, and ex vivo vitreous

409 [20]. GntR represses transcription of the gluconate operon (gntRKPZ) which encodes proteins

410 necessary to utilize gluconate as a carbon source, and repression is relieved by the presence of

411 gluconate [44]. The diarrheal pathogens Vibrio cholera and Escherichia coli are able to adapt to

412 the gastrointestinal tract by utilization of available gluconate [45]. In the absence of gluconate,

413 intraocular expression of $g n t R$ would be expected to repress the expression of the other three

414 genes necessary to metabolize gluconate.

415 The quorum sensing transcriptional regulator gene, $n p r R$, was expressed at significantly

416 higher levels at 8 hours postinfection than at the same time point in BHI. In vitro analysis in our 
417 prior study detected $n p r R$ transcripts during in stationary phase in BHI, LB, and ex vivo vitreous.

418 At the start of stationary phase, NprR regulates PlcR as well as genes involved in cell survival,

419 sporulation, antibiotic resistance, and the metalloprotease nprA gene [46]. In strain ATCC 14579,

$420 n p r R$ is disrupted by a transposon insertion, resulting in a nonfunctional polypeptide. Dubois et

421 al. reported that NprR positively regulates the inhA3 expression [25]. Our results suggest that the

422 absence of inhA3 transcripts in vitro and in vivo was likely due to a nonfunctional NprR in

423 ATCC 14579. The $n p r R$ gene is also repressed by CodY during logarithmic phase growth, an

424 observation consistent with detecting low-level expression at 8 hours in BHI. However, in eye,

$425 n p r R$ expression was 56-fold higher than in BHI, suggesting that expression of $n p r R$ is

426 influenced by in vivo queues.

427 In conjunction with PlcR, $\operatorname{SinR}$ regulates the constitutive expression of $\mathrm{Hbl}$ in $B$.

428 thuringensis biofilms [47]. However, while $h b l$ gene expression was high at the midpoint of

429 infection in our murine model, $\sin R$ transcripts were absent. This is in contrast to high levels in

430 BHI at 8 hours and after growth to stationary phase in BHI, LB, or ex vivo vitreous [20]. We

431 previously observed low levels of expression of $\sin I$, the gene encoding the inhibitor of SinR

432 [48], after growth to stationary phase in BHI and ex vivo vitreous, but not after growth to

433 stationary phase in LB [20]. Similarly, sinI transcripts were not detected in vivo or during

434 logarithmic phase growth in BHI. The absence of $\sin R$ transcripts at 8 hours postinfection again

435 suggests that in vivo, hbl expression might be governed by other factors.

436 Transcripts of entD, the gene encoding the EntD biofilm regulator, were detected at low

437 levels during stationary phase after 18 hours of growth in BHI, LB, and ex vivo vitreous [20], but

438 were not detected at 8 hours in BHI or following intraocular infection. EntD is involved in

439 regulating genes involved in biofilm formation, cell metabolism, cell structure, antioxidation, 
motility, and toxin production [49]. This suggests that EntD might contribute to intraocular

441 survival. However, given the differences between our previous and current findings, low level

442 expression of entD might only occur at later stages of infection and thus the relevance to

443 intraocular infection remains unknown.

444 The PlcR/PapR master quorum sensing regulatory system transactivates a subset of

445 virulence factors at the beginning of stationary phase. Low nutrient conditions and increasing 446 cellular density result in activation of $p l c R$ expression $[31,50]$. Surprisingly, we did not detect

447 transcripts of either the plcR or the papR genes in vivo at 8 hours postinfection. In conjunction

448 with the rapid growth of B. cereus that occurs upon infection of the eye, these results suggest that

449 nutrients are either not limiting, and/or sufficient cell densities have not yet been reached at this

450 time point. plcR expression was observed at the end of the exponential growth phase in LB [51],

451 and given that we previously detected low levels of transcripts in stationary phase after growth in

$452 \mathrm{BHI}, \mathrm{LB}$, and ex vivo vitreous [20], expression of plcR in vivo might occur during narrow

453 window at the end of logarithmic and beginning of stationary phase. Further, plcR expression in

454 the eye during infection is supported by our report that PlcR contributes to inflammation and

455 retinal function loss during experimental B. cereus endophthalmitis [9], and permeability of an in

456 vitro blood retinal barrier [52]. Our in vivo results at this timepoint also suggests that virulence

457 factors controlled by PlcR in vitro might be regulated differently, or respond to different

458 environmental queues during infection.

B. cereus motility is important to its virulence during endophthalmitis [8, 19]. We

460 therefore evaluated expression of genes related to motility and chemotaxis at the midpoint of

461 endophthalmitis. These genes reside in $45 \mathrm{~kb}$ region in the B. cereus ATCC14579 genome [53,

462 54], and, in B. subtilis, are organized in a single operon under the control of CodY [55, 56]. 
463 During stationary phase in ex vivo vitreous, the chemotaxis-related genes cheA, cheY, and cheR,

464 were expressed at low levels, and expression of the genes related to flagellum production and

465 motility fla, fliF, and motB was not detected or detected at only at low levels [20]. In our current

466 study, we detected low levels of cheA transcripts, but did not detect cheY or cheR transcripts at 8

467 hours following infection. However, the motility-related genes $m o t B, f l i F$, and fla expression was

468 higher at this timepoint than after 18 hours of growth in ex vivo vitreous [20]. CheA, CheY, and

469 CheR are involved in switching between tumbling and forward motion in order to adjust to

470 chemoattractant gradients [53, 57-60]. In the eye, nutrients might be homogeneously distributed

471 throughout the vitreous, so following a chemoattractant gradient is not necessary. However,

472 detection of motility-related gene expression at the midpoint of infection supports our previous

473 studies demonstrating the importance of motility to endophthalmitis pathogenesis [8, 19]. These

474 findings suggest that cues necessary for expression of motility genes in the eye were absent from

475 ex vivo vitreous [20].

476 Similar to our findings in ex vivo vitreous [20], the sodA2 gene was the most highly

477 expressed among the genes we evaluated in vivo at 8 hours postinfection. SodA2 is a Mn-based

478 superoxide dismutase [61] that mediates the conversion of superoxide anion $\left(\mathrm{O}_{2}^{-}\right)$and other

479 oxygen radicals to molecular oxygen and hydrogen peroxide $\left(\mathrm{H}_{2} \mathrm{O}_{2}\right)$. These products are then

480 converted into $\mathrm{H}_{2} \mathrm{O}$ and $\mathrm{O}_{2}$ by peroxidases and catalases. B. subtilis SodA provides protection

481 from oxidative stress in growing and sporulating cells [62]. Bacterial SodA might serve as a

482 novel therapeutic target given that human SOD is structurally different [63]. High level

483 expression of the $B$. cereus sodA genes, in particular sodA2, in the eye during infection might

484 interfere with neutrophil function and impede clearance. Indeed, Bacillus replicate seemingly 
without much opposition, even as neutrophils are recruited rapidly and to high numbers into the eye during infection.

$$
\text { An additional target for therapeutic intervention in B. cereus endophthalmitis is the }
$$
surface layer protein, SlpA. We identified SlpA as a significant contributor to disease severity in our murine model of Bacillus endophthalmitis [17]. The mechanism for this effect was linked to

490 activation of nuclear factor kappa-light-chain-enhancer of activated B cells (NF- $\mathrm{B})$ and

491 subsequent inflammatory mediator production from Muller cells [17]. Further investigation

492 revealed that SlpA activated both TLR2 and TLR4 in vitro, and that administration of TLR2/4

493 inhibitors attenuated the severity of infection in our mouse model $(18,24)$. These results

494 suggested that interfering with SlpA-mediated innate immune activation might serve as a novel

495 target for immunomodulatory treatments of $B$. cereus endophthalmitis. Although $\operatorname{slpA}$ transcript

496 levels were not significantly different after 8 hours of growth in vivo or in BHI, detection of

497 transcripts in vivo does support our findings that S-layer protein is present and contributes to the

498 host immune response and poor vision outcomes in a mouse model of Bacillus endophthalmitis. In summary, our results identified the expression of virulence genes and virulence-

500 associated genes at the midpoint of Bacillus infection in the eye, highlighting possible

501 mechanisms for inflammation, retinal damage, and vision deficits in this disease. While our

502 results provide a snapshot of steady state transcript levels at a single, but clinically important

503 time point during infection, these findings lay the groundwork for and demonstrate the feasibility

504 of examining the dynamic B. cereus virulome during the course of endophthalmitis, and further

505 analysis of potential targets for anti-virulence therapies to combat this devastating infection.

\section{Author Statements}


509 PSC, FCM, and MCC designed the study. PSC, FCM, MAE, CL, ALL, and MHM performed the

510 experiments. PSC, FCM, MAE, and CL compiled and analyzed the data. PSC performed the

511 statistical analyses on the data. PSC prepared the original draft of the manuscript. FCM and

512 MCC reviewed and edited the manuscript. PSC and MCC managed the project. MCC acquired

513 the funding for this project. All authors read and approved the final manuscript.

\section{Conflicts of interest}

515 The authors declare that there are no conflicts of interest.

\section{Funding information}

517 This study was supported by NIH Grants R01EY024140, R01EY028810, and R21EY028066 (to

518 MCC), and in part by NIH Grant P30EY027125 (NIH CORE grant to MCC), a Presbyterian Health

519 Foundation Research Support Grant (to MCC), a Presbyterian Health Foundation Equipment Grant

520 (to Robert E. Anderson, OUHSC), and an unrestricted grant to the Dean A. McGee Eye Institute

521 from Research to Prevent Blindness Inc. The funders had no role in study design, data collection

522 and analysis, decision to publish, or preparation of the manuscript.

\section{Acknowledgements}

524 We thank Roger Astley (Department of Ophthalmology, OUHSC) and Mark Dittmar (Dean

525 McGee Eye Institute Animal Facility) for their invaluable technical assistance. We thank Jenny

526 Gipson and Allison Gillaspy at the Laboratory for Molecular Biology and Cytometry Research at

527 OUHSC for assistance and the use of the Core Facility which provided the RNA-Seq service. 
1. Astley RA, Coburn PS, Parkunan SM, Callegan MC. Modeling intraocular bacterial infections. Prog Retin Eye Res 2016;May 3. pii: S1350-9462(16)30023-4.

2. Parkunan SM, Callegan MC. The pathogenesis of bacterial endophthalmitis, p 14-47. Publishing, Cham, Switzerland.

3. Durand ML. Endophthalmitis. Clin Microbiol Infect 2013;19:227-234.

4. Coburn PS, Callegan MC. Endophthalmitis, p 319-340. In Rumelt S (ed), Advances in Ophthalmology, 2012, InTech, Rijeka, Croatia.

JJ, Novosad. Bacterial endophthalmitis: therapeutic challenges and host-pathogen interactions. Prog Retin Eye Res 2007;26:189-203.

6. Callegan MC, Kane ST, Cochran DC, Gilmore MS. Molecular mechanisms of Bacillus endophthalmitis pathogenesis. DNA Cell Biol 2002;21:367-373.

7. Callegan MC, Cochran DC, Kane SJ, Gilmore MS, Gominet M, Lereclus D. 2002;70:5381-5389.

\section{Callegan MC, Kane ST, Cochran DC, Novosad B, Gilmore MS, Gominet M,}


10. Beecher DJ, Olsen TW, Somers EB, Wong ACL. Evidence for contribution of tripartite hemolysin BL, phosphatidylcholine-preferring phospholipase $\mathrm{C}$, and collagenase to virulence of Bacillus cereus endophthalmitis. Infect Immun 2000;68:5269-5276.

11. Callegan MC, Jett BD, Hancock LE, Gilmore MS. Role of hemolysin BL in the pathogenesis of extraintestinal Bacillus cereus infection assessed in an endophthalmitis model. Infect Immun 1999;67:3357-3366.

12. Callegan MC, Booth MC, Jett BD, Gilmore MS. Pathogenesis of gram-positive bacterial endophthalmitis. Infect Immun 1999;67:3348-3356.

13. Budzik JM, Marraffini LA, Schneewind O. Assembly of pili on the surface of Bacillus cereus vegetative cells. Mol Microbiol 2007;66:495-510. layers on the surface of Bacillus cereus strains: phylogenetic origin and ecological pressure. Environ Microbiol 2001;3:493-501.

15. Tagawa Y. Isolation and characterization of flagellar filaments from Bacillus cereus ATCC 14579. Antonie Van Leeuwenhoek 2014;106:1157-1165.

16. Callegan MC, Parkunan SM, Randall CB, Coburn PS, Miller FC, LaGrow AL, Astley RA, Land C, Oh SY, Schneewind O. The role of pili in Bacillus cereus intraocular infection. Exp Eye Res 2017;159:69-76. Sci 2019;60:3727-3739. 

Callegan MC. Bacillus S-Layer-mediated innate interactions during endophthalmitis. Front Immunol 2020; Feb 12;11:215. doi: 10.3389/fimmu.2020.00215. migration in the pathogenesis of Bacillus endophthalmitis. Invest Ophthalmol Vis Sci 2006;47:4461-4467. MC. Expression of Bacillus cereus virulence-related genes in an ocular infection-related environment. Microorganisms 2020; Apr 22;8(4):607. doi: 10.3390/microorganisms8040607. intraocular infection. PLoS One 2014;9(6):e100543. Callegan MC. TLR4 modulates inflammatory gene targets in the retina during Bacillus cereus endophthalmitis. BMC Ophthalmol 2018;18(1):96.

23. Ramadan RT, Ramirez R, Novosad BD, Callegan MC. Acute inflammation and loss of retinal architecture and function during experimental Bacillus endophthalmitis. Curr Eye Res 2006;31:955-965. immune interference attenuates inflammation in Bacillus endophthalmitis. bioRxiv 2020; 06.10.144915; doi: https://doi.org/10.1101/2020.06.10.144915.

\section{Dubois T, Faegri K, Gélis-Jeanvoine S, Perchat S, Lemy C, Buisson C, Nielsen-} LeRoux C, Gohar M, Jacques P, Ramarao N, Slamti L, Kolstø AB, Lereclus D. 
Correction: necrotrophism is a quorum-sensing-regulated lifestyle in Bacillus thuringiensis. PLoS Pathog 2016; Nov 29;12(11):e1006049. doi: 10.1371/journal.ppat.1006049. Erratum for: PLoS Pathog. 2012;8(4):e1002629. characterization of the $h b l A$ gene encoding the B component of hemolysin BL from Bacillus cereus. J Bacteriol 1993;175:6760-6766.

27. Beecher DJ, Pulido JS, Barney NP, Wong AC. Extracellular virulence factors in Bacillus cereus endophthalmitis: methods and implication of involvement of hemolysin BL. Infect Immun 1995;63:632-639. poisoning and food-borne Bacillus cereus strains. J Clin Microbiol 2002;40:3053-3056. Stroman DW. Virulence factor profiles and antimicrobial susceptibilities of ocular Bacillus isolates. Curr Eye Res 2006;31:693-702. in bacteria of the Bacillus cereus group. EMBO J 2002;21:4550-4559. Bacillus cereus potential cell wall peptidase implicated in adhesion, biofilm formation, and virulence. J Bacteriol 2010;192:2638-2642. 

TLR4-dependent retinal gene expression during Bacillus cereus endophthalmitis. Invest Ophthalmol Vis Sci 2016;57:2344.

34. Los FC, Randis TM, Aroian RV, Ratner AJ. Role of pore-forming toxins in bacterial infectious diseases. Microbiol Mol Biol Rev 2013;77:173-207. Wessels MR, Golenbock DT, Malley R. The apoptotic response to pneumolysin is Tolllike receptor 4 dependent and protects against pneumococcal disease. Infect Immun 2005;73:6479-6487.

37. Park JM, Ng VH, Maeda S, Rest RF, Karin M. Anthrolysin O and other gram-positive cytolysins are toll-like receptor 4 agonists. J Exp Med 2004;200:1647-1655.

38. Miyoshi S, Shinoda S. Microbial metalloproteases and pathogenesis. Microbes Infect 2000;2:91-98. Sci 2009;50:4552-4560. envelope-bound metalloprotease (camelysin) from Bacillus cereus is a possible pathogenic factor. Biochim Biophys Acta 2001;1537:132-146. 
41. Grass G, Schierhorn A, Sorkau E, Müller H, Rücknagel P, Nies DH, Fricke B. Camelysin is a novel surface metalloproteinase from Bacillus cereus. Infect Immun 2004;72:219-228. orchestrates the expression of virulence determinants in emetic Bacillus cereus by endophthlamitis. Invest Ophthalmol Vis Sci 2014;55(13):2870.

44. Reizer A, Deutscher J, Saier MH, Reizer J. Analysis of the gluconate (gnt) operon of Bacillus subtilis. Mol Microbiol 1991;5:1081-1089. pathway is obligatory for gluconate utilization and contributes to the pathogenicity of Vibrio cholerae. J Bacteriol 2012;194:3377-3385. Jacques P, Ramarao N, Kolstø AB, Lereclus D. Necrotrophism is a quorum-sensingregulated lifestyle in Bacillus thuringiensis. PLoS Pathog 2012;8(4):e1002629. expression in Bacillus thuringiensis biofilms. PLoS One 2014;9(1):e87532. 

Bacillus cereus EntD as a pivotal protein for the production of numerous virulence factors. Front Microbiol 2015;6:1004. Lereclus D. The PlcR virulence regulon of Bacillus cereus. PLoS One 2008;3:e2793. Bacillus thuringiensis gene that positively regulates transcription of the phosphatidylinositol-specific phospholipase C gene at the onset of the stationary phase. $J$ Bacteriol 1996;178:2749-2756. induces permeability of an in vitro blood-retina barrier. Infect Immun 2008;76(4):13581367. doi:10.1128/IAI.01330-07.

53. Kristoffersen SM, Ravnum S, Tourasse NJ, Okstad OA, Kolsto AB, Davies W. Low concentration of bile salts induce stress responses and reduce motility in Bacillus cereus ATCC 14570. J Bacteriol 2007;189:5302-5313.

\section{Ivanova N, Sorokin A, Anderson I, Galleron N, Candelon B, Kapatral V,} Bhattacharyya A, Reznik G, Mikhailova N, Lapidus A, Chu L, Mazur M, Goltsman E, Larsen N, D'Souza M, Walunas T, Grechkin Y, Pusch G, Haselkorn R, Fonstein M, Ehrlich SD, Overbeek R, Kyrpides N. Genome sequence of Bacillus cereus and comparative analysis with Bacillus anthracis. Nat 2003;423:87-91. 437-452. In Sonenshein AL, Hoch JA, Losick R (ed), Bacillus subtilis and its closest relatives. From genes to cells, 2002, ASM Press, Washington, D.C. 

Environmental regulation of Bacillus subtilis sigma(D)-dependent gene expression. $J$ Bacteriol 2000;182:3055-3062. transcriptomic analyses of mildly and severely salt-stressed Bacillus cereus ATCC 14579 cells. Appl Environ Microbiol 2009;75:4111-4119.

58. Barak R, Eisenbach M. Acetylation of the response regulator, CheY, is involved in bacterial chemotaxis. Mol Microbiol 2001;40:731-743.

59. Ramakrishnan R, Schuster M, Bourret RB. Acetylation at Lys-92 enhances signaling by the chemotaxis response regulator protein CheY. Proc Natl Acad Sci U S A 1998;95:4918-4923.

60. Wolfe AJ, Conley MP, Berg HC. Acetyladenylate plays a role in controlling the direction of flagellar rotation. Proc Natl Acad Sci U S A 1988;85:6711-6715.

61. Stroupe ME, DiDonato M, Tainer JA. Manganese Superoxide Dismutase. In

62. Inaoka T, Matsumura Y, Tsuchido T. SodA and manganese are essential for resistance to oxidative stress in growing and sporulating cells of Bacillus subtilis. J Bacteriol 1999;181:1939-1943. superoxide dismutase. Acta Crystallogr Sect F Struct Biol Cryst Commun 2007;63:10031007. 
bioRxiv preprint doi: https://doi.org/10.1101/2020.07.02.184630; this version posted July 2, 2020. The copyright holder for this preprint (which was not certified by peer review) is the author/funder. All rights reserved. No reuse allowed without permission.

708 Table 1. Mean number of reads and percentage of reads that mapped to the $\boldsymbol{B}$. cereus

709 ATCC 14579 genome for each environmental condition.

\begin{tabular}{ccc}
\hline Environment & Mean Number of Reads & Percentage of Reads Mapped \\
\hline BHI & $25,505,762$ & $96 \%$ \\
In vivo & $12,360,200$ & $99 \%$ \\
\hline
\end{tabular}


FIGURE LEGENDS

712 Figure 1. Analysis of the growth of B. cereus in BHI and in vivo. Growth of B. cereus in BHI

713 (Brain Heart Infusion) 8 hours after back dilution, and in vivo 8 hours postinfection. The CFU/ml

714 of $B$. cereus 8 hours after back dilution was significantly higher than the concentration in mouse

715 eyes 8 hours after infection $(* \mathrm{p}=0.0038)$. The mean $\mathrm{CFU} / \mathrm{ml} \pm$ the standard error of the mean of

7166 independent BHI cultures, and the mean CFU/eye \pm standard error of the means of 5 tubes

717 (each tube containing 5 eyes) and 3 independent experiments are shown.

718 Figure 2. Principal component analysis of B. cereus gene expression in BHI or in vivo.

719 The expression levels of genes related to virulence, regulation of virulence, chemotaxis, and

720 motility from each of the three independent RNA-Seq runs of B. cereus cultivated in BHI 8

721 hours after back-dilution or 8 hours following intravitreal infection of mouse eyes was analyzed

722 by principal component analysis. Gene expression levels in BHI from each of the 3 independent

723 RNA-Seq experiments clustered distinctly from gene expression levels in infected mouse eyes

724 from each of the 3 independent RNA-Seq experiments.

725 Figure 3. Normalized virulence-related gene expression in BHI or in vivo. Reads per kilobase

726 per million (RPKM) for the hemolysin BL genes hblL1 (*p=0.0057), hblL2 (**p=0.0173), and

727 hblB $(* * * \mathrm{p}=0.0096)$ in vivo relative to BHI (A); nonhemolytic enterotoxin genes nheL1

$728 \quad(* \mathrm{p}<0.0001)$ and nheL2 (ns = not significant) in vivo relative to $\mathrm{BHI}(\mathrm{B})$; the enterotoxin genes

729 entA (* $\left.{ }^{*}=0.0068\right)$, entC (ns), and the entFM (ns) in vivo relative to BHI (C); the hlyA (ns) and

$730 \operatorname{cerO}(* \mathrm{p}=0.0050)$ genes in vivo relative to BHI (D); and the metalloprotease inhA1 $(* \mathrm{p}=0.0270)$,

731 inhA2 (ns), inhA3 (not detected), and calY (not detected) genes in vivo relative to BHI (E). BHI

732 is shown in red, and in vivo in blue. RPKM values are the means \pm the standard deviations of

733 three independent RNA-Seq runs. 
734 Figure 4. Normalized transcriptional regulatory gene expression in BHI or in vivo. Reads

735 per kilobase per million $(\mathrm{RPKM})$ for the transcriptional regulator genes $\sin R\left({ }^{*} \mathrm{p}=0.0024\right)$ in

736 BHI relative to in vivo, and $\sin I$ (not detected) (A); the genes entD (not detected) and $\operatorname{cod} Y$

$737 \quad(* \mathrm{p}=0.0196)$ in BHI relative to in vivo $(\mathrm{B})$; the genes gntR (ns = not significant) and $n p r R$

$738\left({ }^{*} \mathrm{p}=0.0014\right)$ in vivo relative to $\mathrm{BHI}(\mathrm{C})$; and the genes plcR (ns) and papR $\left({ }^{*} \mathrm{p}=0.0025\right)$ in BHI

739 relative to in vivo (D). BHI is shown in red, and in vivo in blue. RPKM values are the means \pm

740 the standard deviations of three independent experiments RNA-Seq runs.

741 Figure 5. Normalized motility and chemotaxis-related gene expression in BHI or in vivo.

742 Reads per kilobase per million (RPKM) for the motility-related genes fla $(* \mathrm{p}<0.0001)$, fliF

$743(* * \mathrm{p}=0.0064)$, and $\operatorname{motB}(* * * \mathrm{p}=0.0147)$ in $\mathrm{BHI}$ relative to in vivo $(\mathrm{A})$; and the chemotaxis-

744 related genes $\operatorname{cheA}\left({ }^{*} \mathrm{p}=0.0038\right), \operatorname{cheY}\left({ }^{* *} \mathrm{p}=0.029\right)$, and $\operatorname{cheR}(* * * \mathrm{p}=0.0415)$ in BHI relative to

745 in vivo (B). BHI is shown in red, and in vivo in blue. RPKM values are the means \pm the standard

746 deviations of three independent RNA-Seq runs.

747 Figure 6. Normalized superoxide dismutase (SodA) gene expression in BHI or in vivo.

748 Reads per kilobase per million (RPKM) for the genes sodA1 (ns = not significant) and sodA2

749 (*p=0.0460) in vivo relative to BHI. BHI is shown in red, and in vivo in blue. RPKM values are

750 the means \pm the standard deviations of three independent RNA-Seq runs.

751 Figure 7. Normalized surface layer protein (SIpA) gene expression in BHI or in vivo. Reads

752 per kilobase per million $(\mathrm{RPKM})$ for the $\operatorname{slpA}$ gene $(\mathrm{ns}=$ not significant) in vivo relative to BHI.

$753 \mathrm{BHI}$ is shown in red, and in vivo in blue. RPKM values are the means \pm the standard deviations

754 of three independent RNA-Seq runs. 
755 Figure 1. Analysis of the growth of B. cereus in BHI and in vivo.

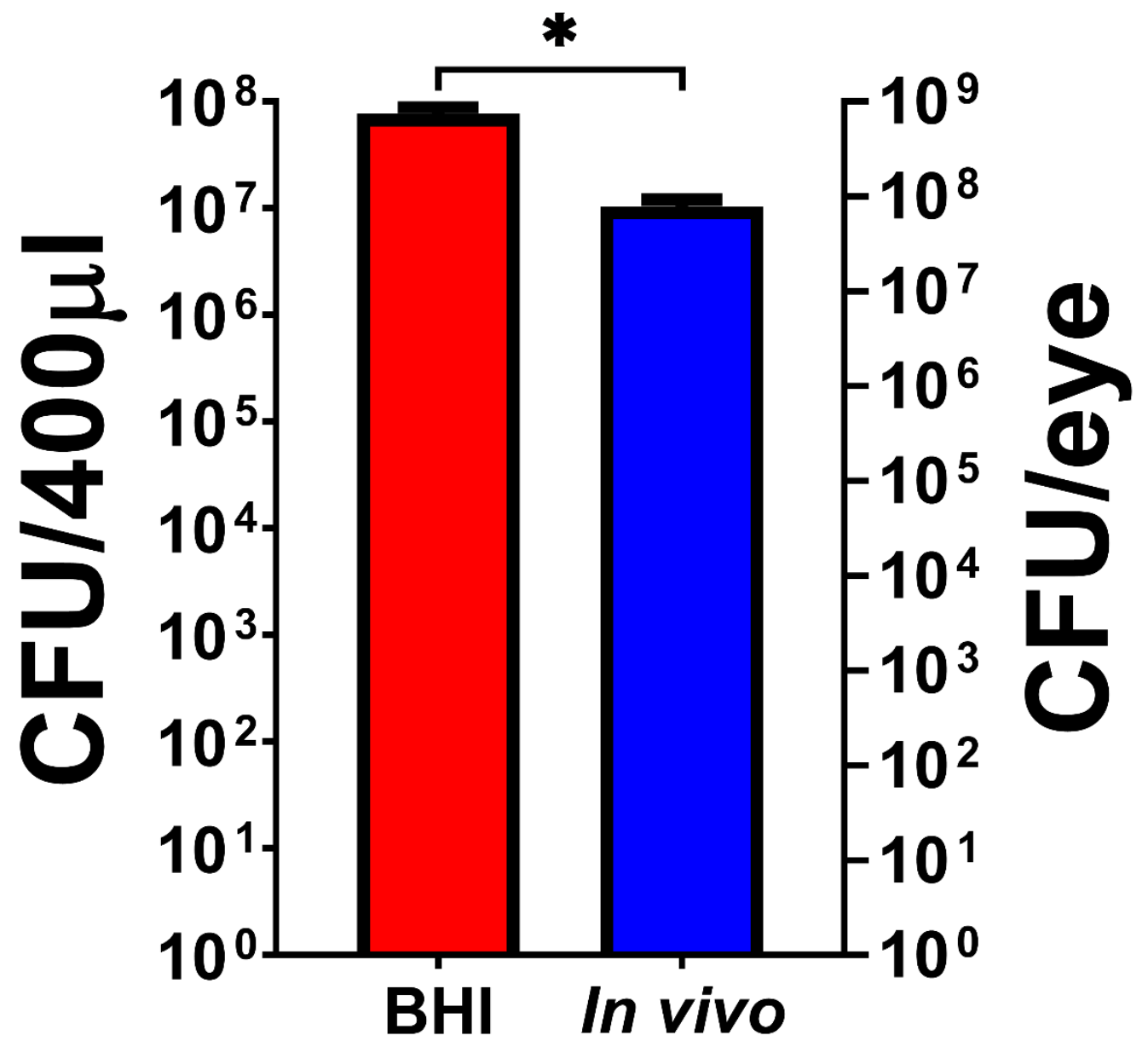


bioRxiv preprint doi: https://doi.org/10.1101/2020.07.02.184630; this version posted July 2, 2020. The copyright holder for this preprint (which was not certified by peer review) is the author/funder. All rights reserved. No reuse allowed without permission.

757 Figure 2. Principal component analysis of B. cereus gene expression in BHI or in vivo.

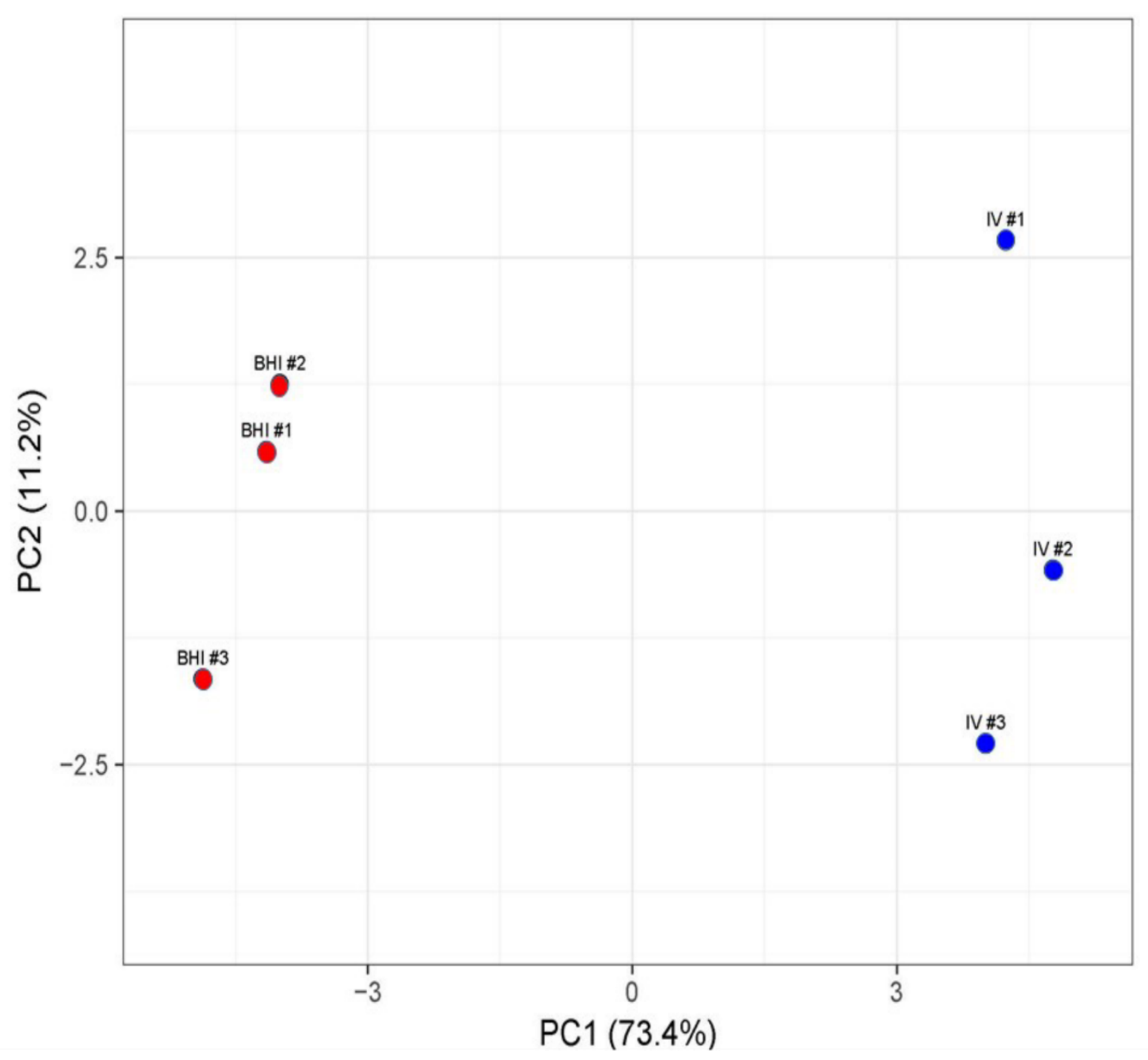


Figure 3. Normalized virulence-related gene expression in BHI or in vivo.

(A) Enterotoxins (hbIL1, hblL2, hbIB)

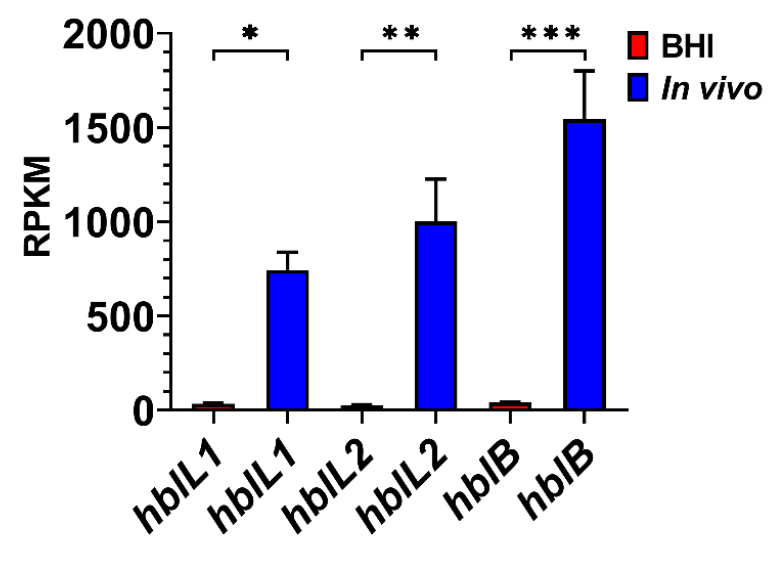

(C) Enterotoxins (entA, C, FM)

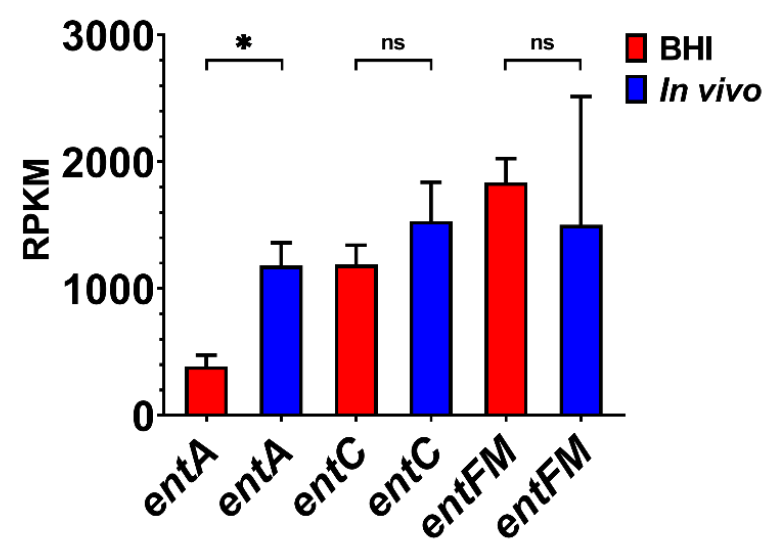

(B) Enterotoxins (nheL1, L2)

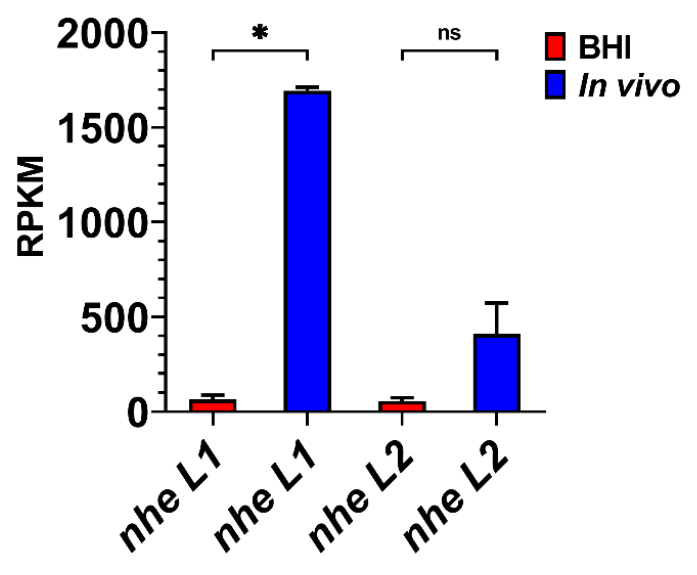

(D) Hemolytic Toxins

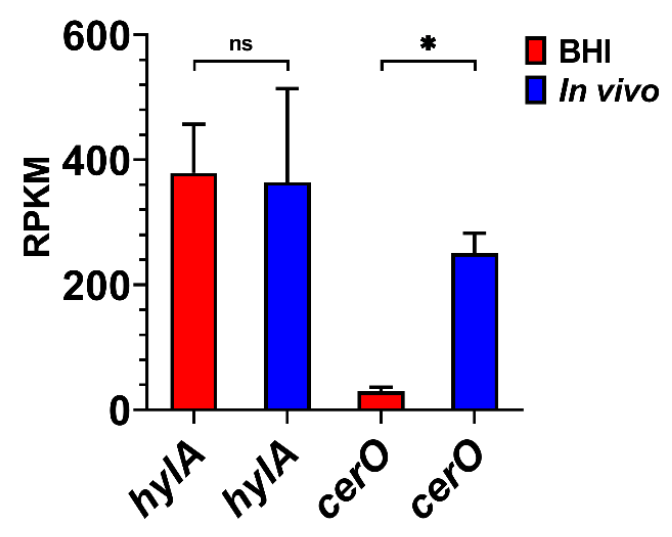

(E) Metalloproteases

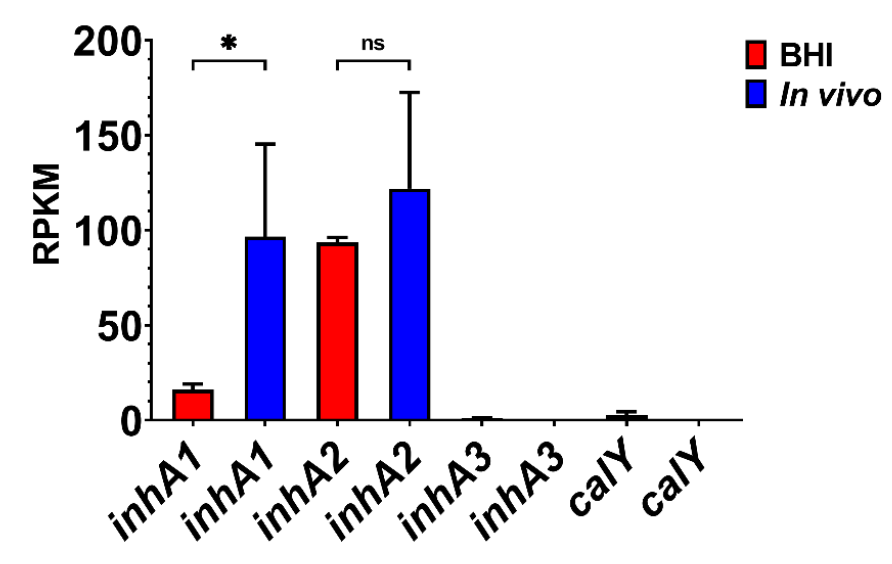


761 Figure 4. Normalized transcriptional regulatory gene expression in BHI or in vivo.

(A) $\sin R / \operatorname{sinl}$

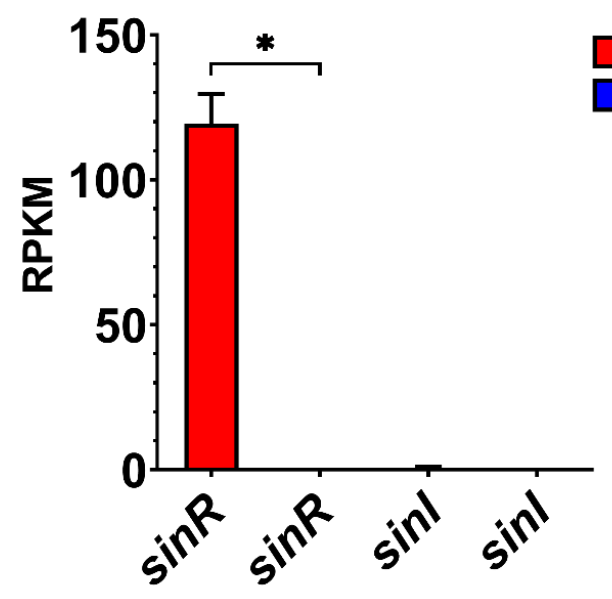

(C)

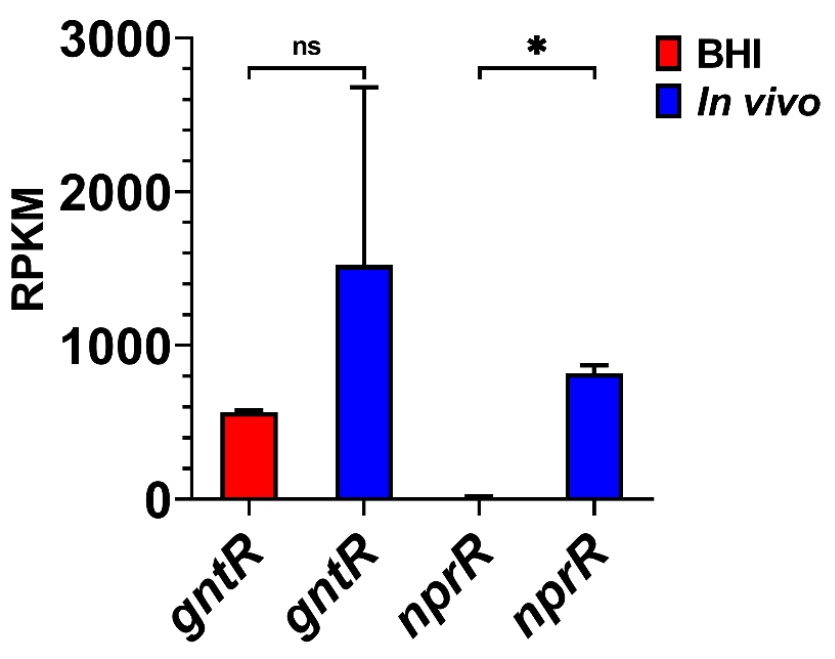

(B) entD/codY

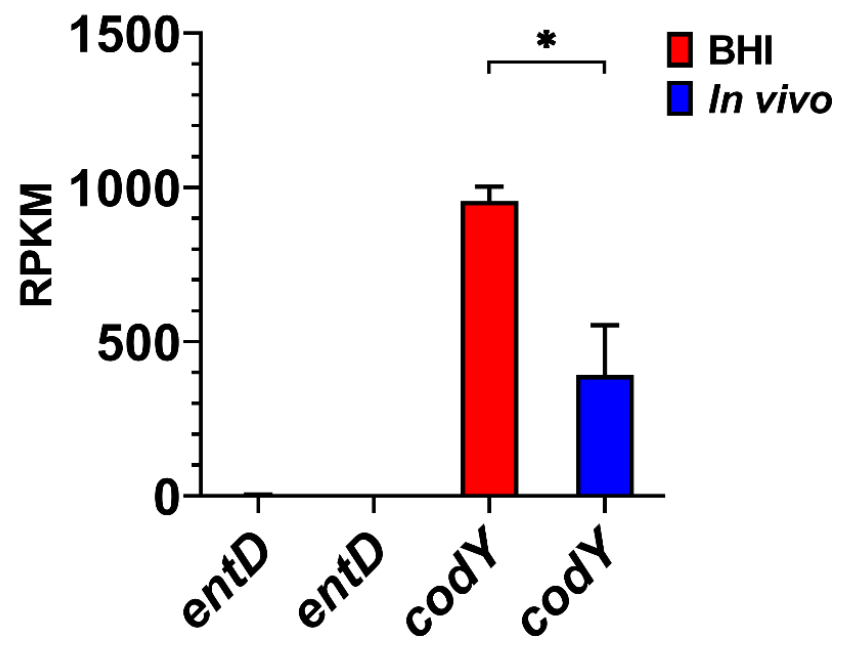

(D) plcR/papR

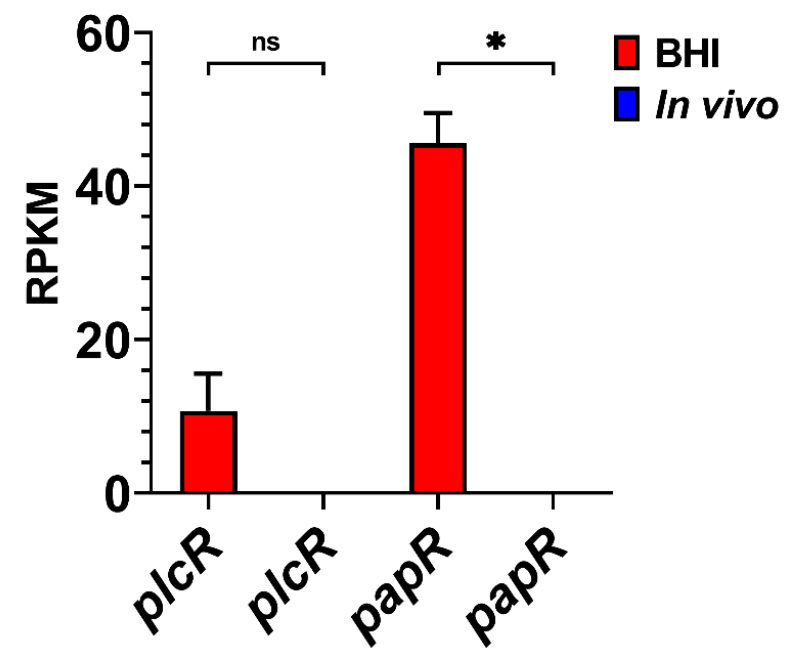


763 Figure 5. Normalized motility and chemotaxis-related gene expression in BHI or in vivo.

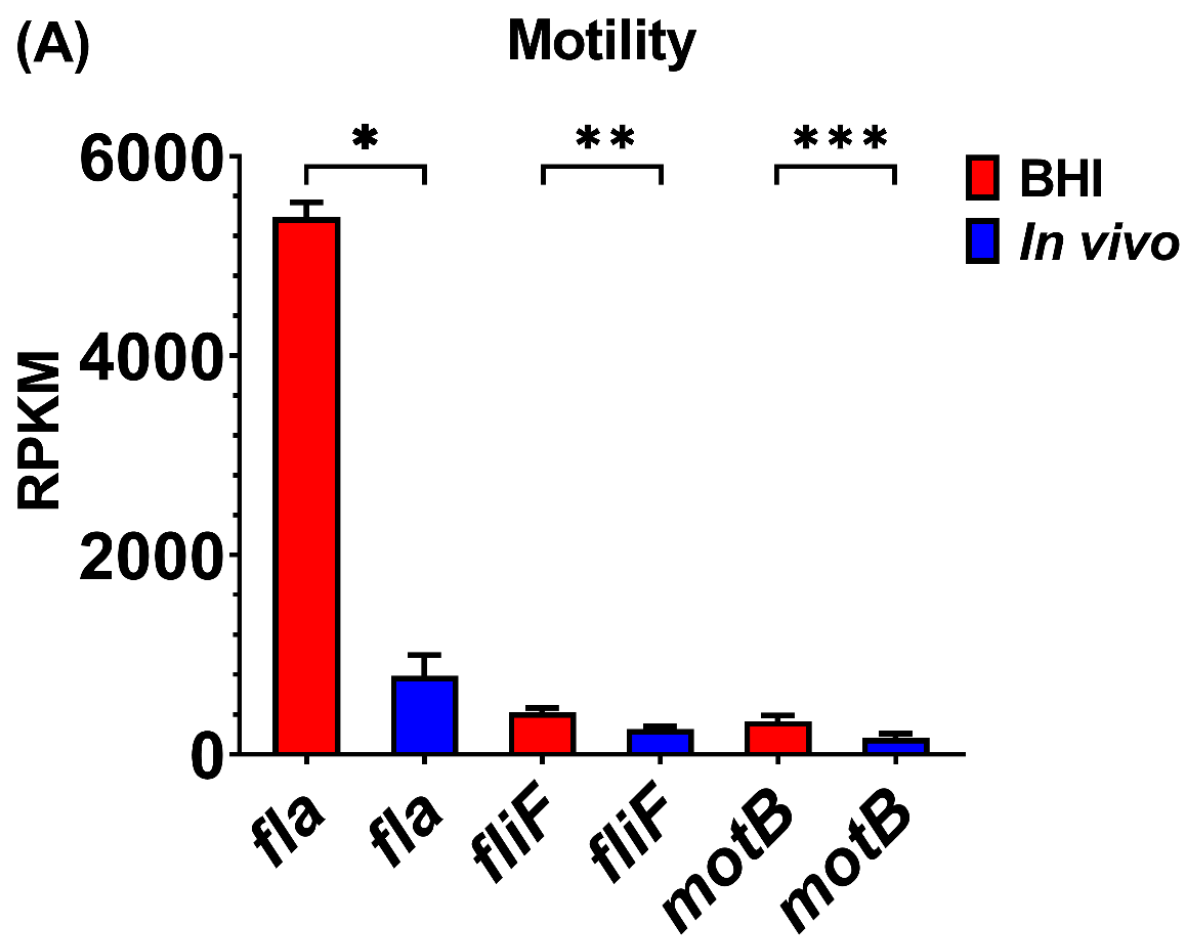

(B) Chemotaxis

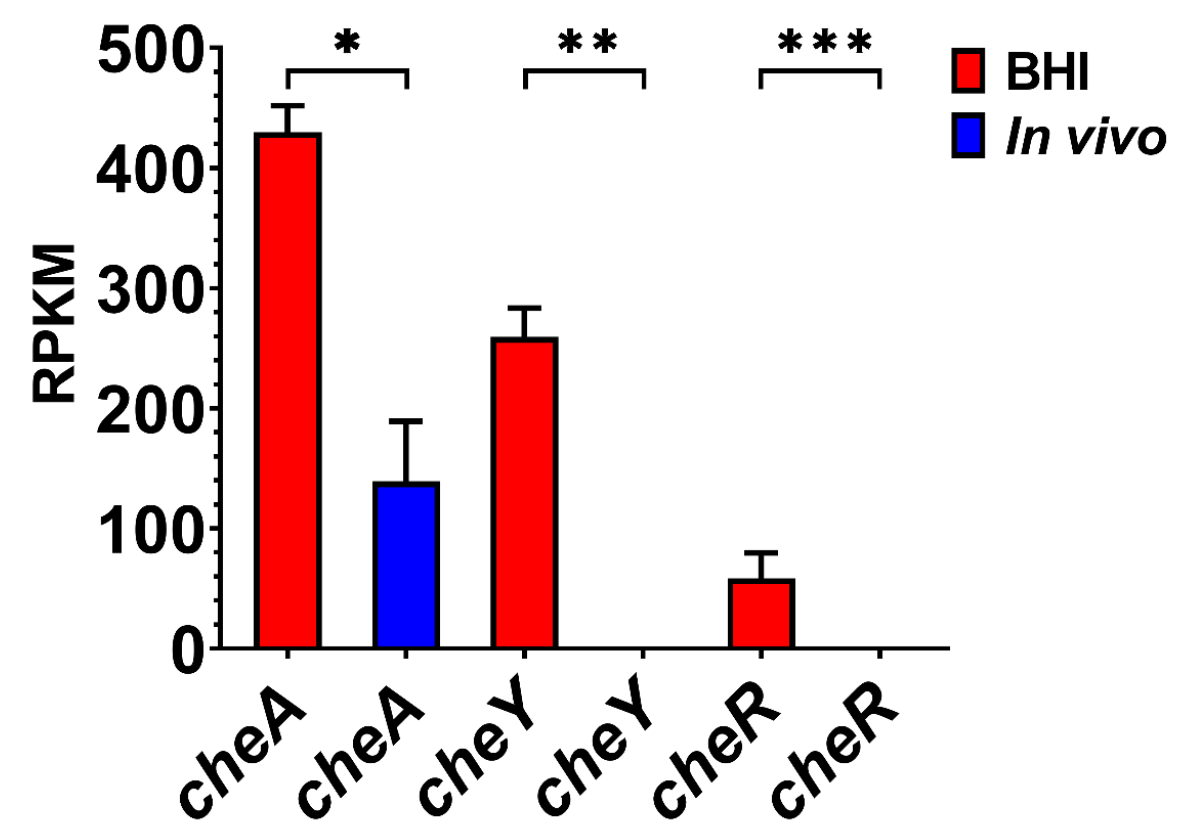


765 Figure 6. Normalized superoxide dismutase gene expression in BHI or in vivo.

\section{SOdA}

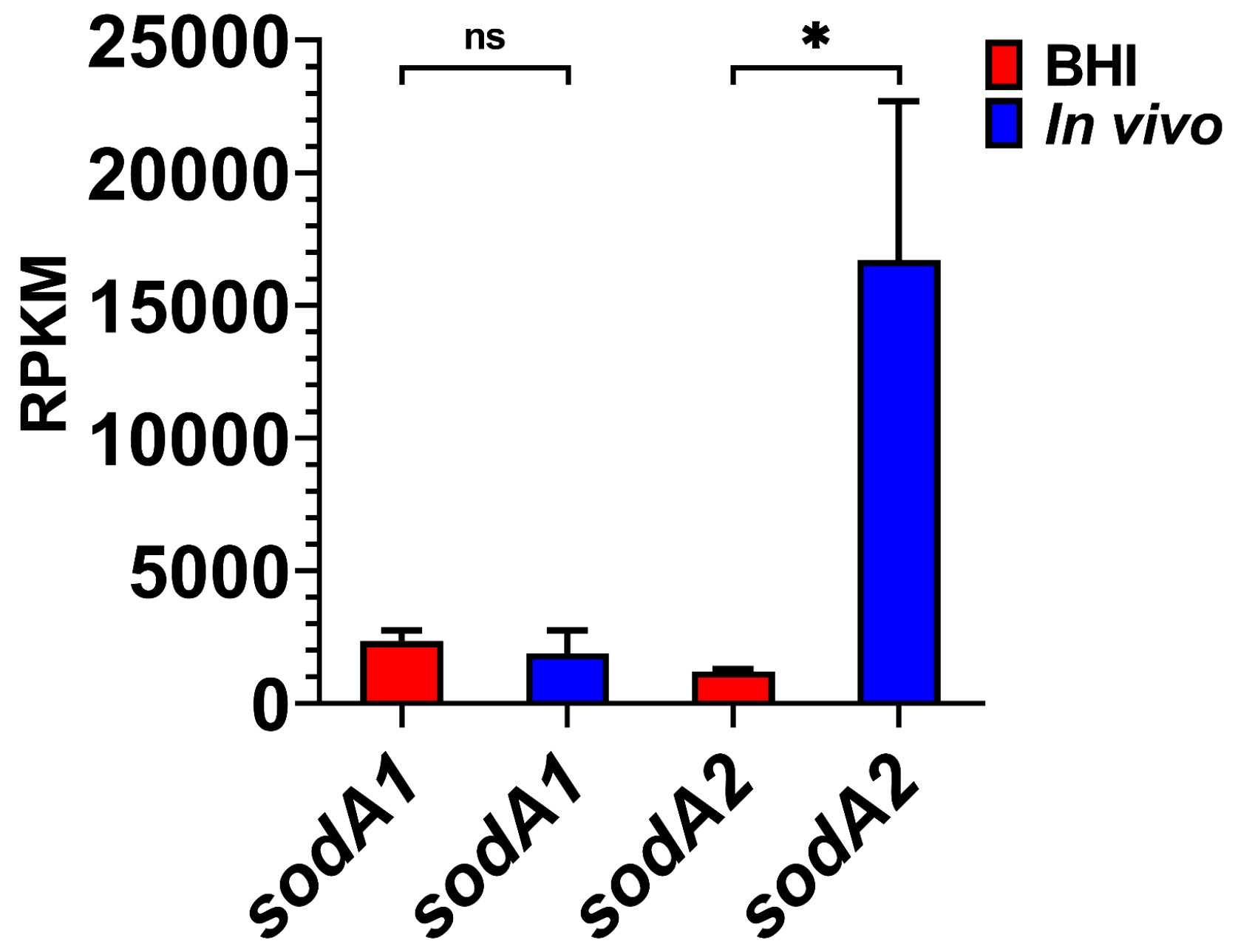


767 Figure 7. Normalized surface layer protein (SlpA) gene expression in BHI or in vivo.

$$
\text { slpA }
$$

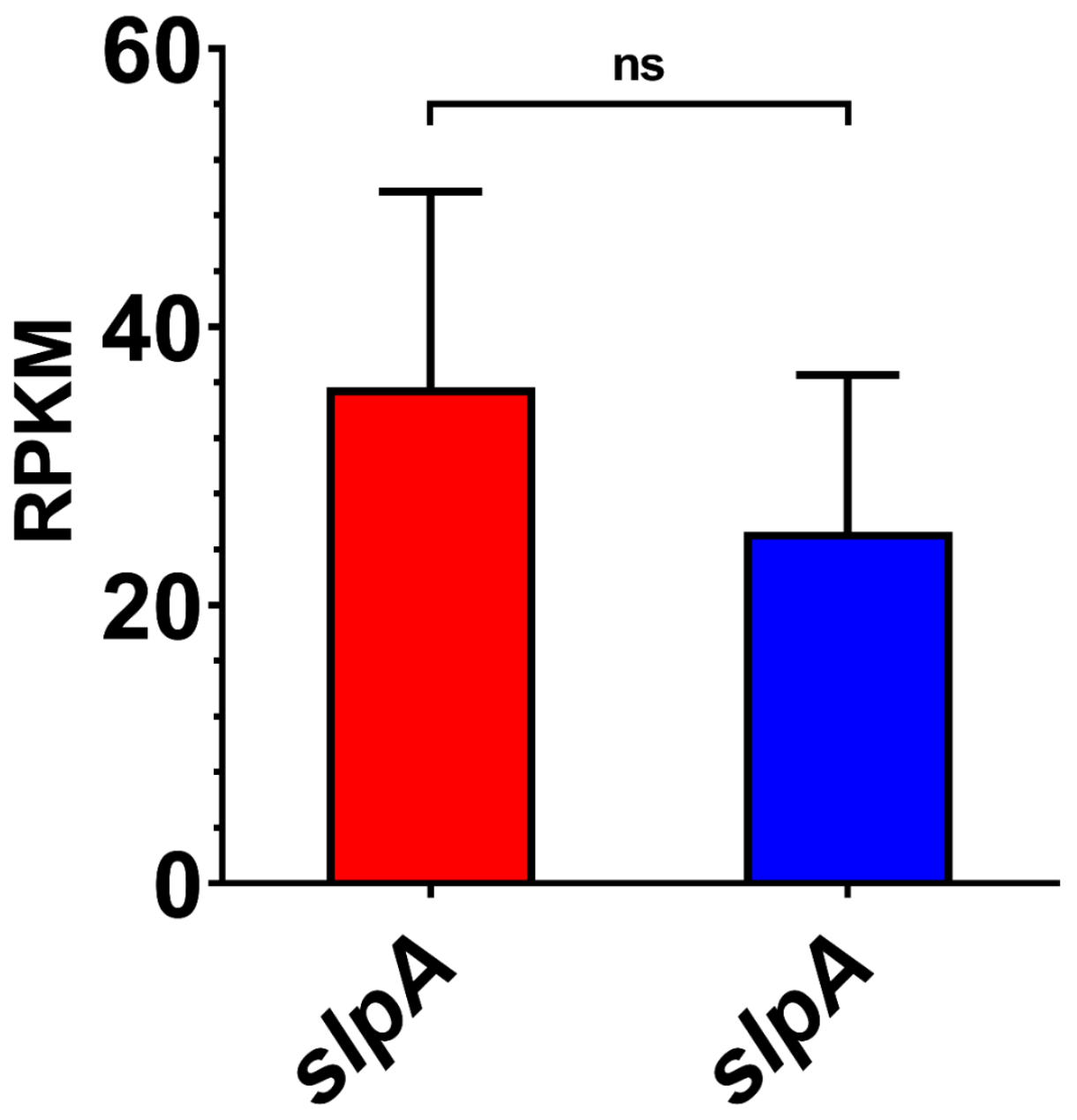

